\title{
Obesity prevalence and its relation with some sociodemographic factors in bank employee of Aurangabad city, Maharashtra, India
}

\author{
Salma Hirani ${ }^{1}$, Bina M. Kuril ${ }^{1}$, Deepak K. Lone ${ }^{2 *}$, R. T. Ankushe ${ }^{1}$, Mohan K. Doibale ${ }^{1}$
}

\begin{abstract}
${ }^{1}$ Department of Community Medicine, Government Medical College, Aurangabad, Maharashtra, India
${ }^{2}$ Medical Officer, Assistant Director of Health Services, Leprosy, Jalna, Maharashtra, India
\end{abstract}

Received: 22 April 2016

Accepted: 16 May 2016

\section{*Correspondence:}

Dr. Deepak K. Lone,

E-mail:dpklone@gmail.com

Copyright: (c) the author(s), publisher and licensee Medip Academy. This is an open-access article distributed under the terms of the Creative Commons Attribution Non-Commercial License, which permits unrestricted non-commercial use, distribution, and reproduction in any medium, provided the original work is properly cited.

\section{ABSTRACT}

Background: India is currently experiencing rapid epidemiological transition with rising prevalence of obesity which may be due to increasing adoption of sedentary lifestyle and changing dietary pattern of the urban population. Certain occupations predispose individuals to sedentary lifestyles and some of these are white collar jobs characterized by sitting for long periods of time like financial institutions (banks). The aim and objectives of the study was to know the prevalence of obesity in bank employees and to study associated socioeconomic factors.

Methods: A population based cross sectional study was conducted. Banks were listed according to sectors i.e. government, co-operative and private. The banks were selected randomly from these three sectors using stratified proportionate random sampling technique. The study participants were interviewed by the investigator with the help of predesigned proforma which included information about demographic \& socio-economic data, medical and family history, clinical examination, and anthropometric measurements (height, weight, waist and hip circumference).

Results: According to BMI classification for Asian population, $47.9 \%$ and $29.6 \%$ bank employees were found to be overweight and obese (GO) respectively. Whereas $65.0 \%$ bank employees had obesity by waist-hip ratio and only $6.25 \%$ bank employees had isolated generalized obesity. Overall $17.95 \%$ bank employees had isolated abdominal obesity and $44.6 \%$ bank employees had combined obesity.

Conclusions: The prevalence of generalized as well abdominal obesity was very high among bank employees. The associated risk factors which were found to be significant were age, female gender, higher socioeconomic status, and higher employees' posts.

Keywords: Bank employee, Obesity, BMI, Urban area, Abdominal obesity

\section{INTRODUCTION}

Obesity is one of the most important public health problems of global significance. ${ }^{1}$ The worldwide prevalence of obesity nearly doubled between 1980 and 2014. In 2014, 39\% of adults aged 18 years and older were overweight and $11 \%$ of men and $15 \%$ of women worldwide were obese. Thus, about 2 billion people are overweight and more than half a billion adults worldwide are obese. ${ }^{2,3}$ Overweight and obesity are estimated to account for 3.4 million deaths per year and 93.6 million DALYs. ${ }^{4}$

India, with 1.2 billion people is the second most populous country in the world and is currently experiencing rapid epidemiological transition. There is rising prevalence of obesity in India. ${ }^{5-8}$ The percentage of overweight and obesity increased from $11 \%$ in national family health survey (NFHS-2) to $15 \%$ in NFHS-3 and more than thrice in urban areas. This may be due to increasing 
adoption of sedentary lifestyle and changing dietary pattern of the urban population. ${ }^{9}$

Certain occupations predispose individuals to sedentary lifestyles and some of these are white collar jobs characterized by sitting for long periods of time. Workers of financial institutions (banks) who serve in the banking hall (tellers and customer service personnel) and back office, spend the greater part of the day sitting down due to the nature of their work. ${ }^{10}$ Therefore, workers in such institutions become susceptible to developing obesity and or overweight which could predispose them to chronic diseases associated with physical inactivity. ${ }^{11-13}$

Keeping all these in mind a population based cross sectional study was conducted to know the prevalence of obesity in bank employees of the urban area.

The aim of study was to estimate the prevalence of obesity among bank employees and study its relation with some sociodemographic factors.

\section{METHODS}

The present population based cross-sectional study was carried out in urban bank employees from April 2014 to September 2014. The study was approved by institutional ethics committee of Government Medical College \& Hospital, Aurangabad, India.

Considering the prevalence of obesity of $16 \%$, and precision of $5 \%$, the required sample size is calculated with the help of this formula: ${ }^{5}$

$$
\text { Sample size }(\mathrm{N})=\mathrm{P} \times \mathrm{Q} \times \mathrm{Z}^{2} / \mathrm{L}^{2}
$$

Where, $\mathrm{P}=$ Prevalence of obesity $=16 \%, \mathrm{Q}=100-\mathrm{P}=$ $100-16=84 \%, Z=2$ (at 95\% Confidence Interval) and $\mathrm{L}$ $=$ Precision $=5 \%$. Calculated sample size came to 216 . Assuming non response rate of about $10 \%$, a sample size of 240 was taken for study.

Banks were listed according to sectors i.e. government, co-operative and private. The banks were selected randomly from these three sectors using stratified proportionate random sampling technique. The pilot study was conducted during March 2014 in two banks selected randomly, to check the predesigned proforma and to know the feasibility of the study. Prior permission was sought from the regional offices for the conduction of interview and health check-up on the fixed days.

Selected bank and/or its branches were visited on the appointed fixed days. The administrative authorities of selected banks were explained the nature and purpose of this study and permission taken to carry out the study.

Employees who had at least 5 years of working experience in the banks were included in the study. Those who were working for more than 5 years in the banks were excluded. Pregnant employees, employees absent on the days of the interview, persons who were on antidepressant drugs or taking steroids and employees not willing to participate in the study were also excluded. All employees eligible as per inclusion and exclusion criteria were selected and after obtaining their written informed consent, interview and clinical examination of bank employees were conducted by the investigator.

The study participants were interviewed by the investigator with the help of predesigned proforma which included information about demographic \& socioeconomic data, medical and family history, clinical examination, and anthropometric measurements (height, weight, waist and hip circumference).

Once the demographic and socioeconomic information filled in the proforma, detailed clinical examination was done including general and systemic examinations. Weight of the study participant was taken by standardized digital weighing machine up to accuracy of $0.1 \mathrm{~kg}$. Height was measured without footwear in $\mathrm{cm}$ by using metric scale on walls up to accuracy of $0.1 \mathrm{~cm}$. BMI was calculated as weight in kilograms divided by the square of the height in meters $\left(\mathrm{kg} / \mathrm{m}^{2}\right)$. Subjects were classified according to WHO Classification based on BMI. ${ }^{14}$ Waist circumference (WC) was measured at the midpoint between the lower margin of the least palpable rib and the top of the iliac crest in the mid axillary line, using a stretch-resistant tape. Hip circumference (HC) was measured around the widest portion of the buttocks, with the tape parallel to the floor. Waist circumference $>90 \mathrm{~cm}$ in males and $>80 \mathrm{~cm}$ in females were the cut off levels for subjects with abdominal obesity. ${ }^{15}$ Waist-Hip ratio (WHR) was also calculated and classified with criteria WHR $>0.90$ for males and $>0.85$ for females. ${ }^{15}$ The occupation and the SES was assessed as per modified Kuppuswamy's socioeconomic status scale (2014) ${ }^{16}$.

\section{Definitions of obesity ${ }^{5,14}$}

\section{Overweight}

It is defined as a BMI $\geq 23 \mathrm{~kg} / \mathrm{m}^{2}$ but $<25 \mathrm{~kg} / \mathrm{m}^{2}$ for both genders (based on the World Health Organization Asia Pacific Guidelines) with or without abdominal obesity (AO).

\section{Generalized obesity $(G O)$}

It is defined as a BMI $\geq 25 \mathrm{~kg} / \mathrm{m}^{2}$ for both genders (based on the World Health Organization Asia Pacific Guidelines) with or without abdominal obesity (AO) and shortly labelled as obese.

\section{Abdominal obesity (AO)}

It is defined as a waist circumference (WC) $\geq 90 \mathrm{~cm}$ for men and $\geq 80 \mathrm{~cm}$ for women with or without generalized obesity (GO). 
Obesity by waist-hip ratio (WHR)

It is defined as WHR $>0.90 \mathrm{~cm}$ in males and WHR $>0.85$ $\mathrm{cm}$ in females.

\section{Isolated generalized obesity (IGO)}

It is defined as BMI $\geq 25 \mathrm{~kg} / \mathrm{m}^{2}$ with waist circumference of $<90 \mathrm{~cm}$ in men and $<80 \mathrm{~cm}$ in women.

\section{Isolated abdominal obesity (IAO)}

It is defined as a waist circumference of $\geq 90 \mathrm{~cm}$ in men or $\geq 80 \mathrm{~cm}$ in women with a BMI $<25 \mathrm{~kg} / \mathrm{m}^{2}$.

\section{Combined obesity ( $\mathrm{CO})$}

It is defined as individuals with both generalized obesity (GO) and abdominal obesity (AO).

All study participants were advised about daily exercise and healthy diet. Those found to be suffering from any disease were referred to tertiary care centre for further management.

\section{Data analysis and statistics}

Qualitative/categorical data was presented as numbers and percentages. Chi square test and Fisher's exact test were used as a test of significance for qualitative data. Quantitative data was presented as mean and standard deviations. Student's t test and ANOVA were used to compare mean difference among groups for normally distributed continuous variables (e.g. age). Significant difference was considered when $\mathrm{p}$ value was less than 0.05. All Data were first entered in Microsoft Excel 2007 and analysed using SPSS Version20.0.

\section{RESULTS}

Out of 240 bank employees in this study, 100 (41.7\%) were from government bank and $46(19.1 \%)$ employees were from co-operative banks followed by 94 (39.2\%) were from private bank sectors. There were $200(83.3 \%)$ male and $40(16.7 \%)$ female bank employees in the study. Total $45.0 \%$ bank employees were in the age group of 45 and above years. Considering religion, $75.4 \%$ of bank employees were Hindu followed by $15.4 \%$ belonging to Buddhism, $6.3 \%$ of other religions and only few $(2.9 \%)$ bank employees were of Muslim religion in the study (Table 1).

Among total bank employees $42 \%$ were educated up to intermediate or post high school diploma followed by graduation or post-graduation $(26.3 \%)$. Only $12.1 \%$, $15.4 \%$ and $4.2 \%$ bank employees were educated to primary/middle school, high school and professional or honours respectively. Bank employees working as managers, officers, clerks, and attendants were $20.0 \%$,
$18.3 \%, 34.2 \%$ and $27.5 \%$ respectively in this study (Table 1).

Table 1: Socio-demographic characteristics of bank employees.

\begin{tabular}{|c|c|c|c|}
\hline & Characteristics & $\begin{array}{l}\text { Frequency } \\
(\mathrm{N}=240)\end{array}$ & Percent \\
\hline \multirow{3}{*}{$\begin{array}{l}\text { Bank } \\
\text { sector }\end{array}$} & Government banks & 100 & 41.7 \\
\hline & Cooperative banks & 46 & 19.1 \\
\hline & Private banks & 94 & 39.2 \\
\hline \multirow{2}{*}{ Gender } & Male & 200 & 83.3 \\
\hline & Female & 40 & 16.7 \\
\hline \multirow{3}{*}{$\begin{array}{l}\text { Age } \\
\text { groups }\end{array}$} & 25 to 34 & 90 & 37.5 \\
\hline & 35 to 44 & 42 & 17.5 \\
\hline & 45 and above & 108 & 45.0 \\
\hline \multirow{4}{*}{ Religion } & Hindu & 181 & 75.4 \\
\hline & Muslim & 7 & 2.9 \\
\hline & Buddhism & 37 & 15.4 \\
\hline & Others & 15 & 6.3 \\
\hline \multirow{5}{*}{ Education } & $\begin{array}{l}\text { Primary/Middle } \\
\text { school }\end{array}$ & 29 & 12.1 \\
\hline & High school & 37 & 15.4 \\
\hline & $\begin{array}{l}\text { Intermediate or post } \\
\text { high school diploma }\end{array}$ & $\begin{array}{ll}\mathrm{t} & 101\end{array}$ & 42.1 \\
\hline & $\begin{array}{l}\text { Graduate or post } \\
\text { graduate }\end{array}$ & 63 & 26.3 \\
\hline & $\begin{array}{l}\text { Professional or } \\
\text { honours }\end{array}$ & 10 & 4.2 \\
\hline \multirow{4}{*}{$\begin{array}{l}\text { Bank } \\
\text { employees } \\
\text { posts }\end{array}$} & Managers & 48 & 20.0 \\
\hline & Officers & 44 & 18.3 \\
\hline & Clerks & 82 & 34.2 \\
\hline & Attendants & 66 & 27.5 \\
\hline \multirow{4}{*}{$\begin{array}{l}\text { Socio- } \\
\text { economic } \\
\text { status }\end{array}$} & Upper (I) & 51 & 21.3 \\
\hline & Upper middle (II) & 123 & 51.3 \\
\hline & Lower middle (III) & 66 & 27.5 \\
\hline & Total & 240 & 100 \\
\hline
\end{tabular}

Distribution of generalized obesity in bank employees according to BMI classification for Asian population shows that $47.9 \%$ and $29.6 \%$ bank employees were found to be overweight and obese $(\mathrm{GO})$ respectively whereas only $18.8 \%$ were in normal BMI range and very few (3.8\%) bank employees were underweight (Table 2).

Table 2: Prevalence of generalized obesity (GO) in bank employees.

\begin{tabular}{|lll|}
\hline $\begin{array}{l}\text { BMI classification (for } \\
\text { Asians) in bank employees }\end{array}$ & Frequency & Percentage \\
\hline Underweight $(<18.5)$ & 9 & 3.8 \\
\hline Normal $(18.5-22.9)$ & 45 & 18.8 \\
\hline Overweight $(23.0-24.9)$ & 115 & 47.9 \\
\hline Obese $(>25.0)$ & 71 & 29.6 \\
\hline Total & $\mathbf{2 4 0}$ & $\mathbf{1 0 0 . 0}$ \\
\hline
\end{tabular}

BMI classification for Asian population was used for defining generalized obesity. 
Overall $62.5 \%$ bank employees had abdominal obesity in our study. Whereas $65.0 \%$ bank employees had obesity by waist-hip ratio and only $6.25 \%$ bank employees had isolated generalized obesity. Overall $17.95 \%$ bank employees had isolated abdominal obesity and $44.6 \%$ bank employees had combined obesity (Table 3).

Table 3: Prevalence of various types of obesity in bank employees.

\begin{tabular}{|lllll|}
\hline Type of obesity & \multicolumn{2}{c|}{ Present } & \multicolumn{2}{l|}{ Absent } \\
\hline & No & $\%$ & No & $\%$ \\
\hline AO & 150 & 62.5 & 90 & 37.5 \\
\hline Obesity by WHR & 156 & 65 & 84 & 35 \\
\hline IGO) & 15 & 6.25 & 225 & 93.75 \\
\hline IAO) & 43 & 17.95 & 197 & 82.05 \\
\hline Combined obesit & 107 & 44.6 & 133 & 55.4 \\
\hline
\end{tabular}

The difference in age wise distribution of generalised obesity in bank employees was not found to be statistically significant $[\mathrm{p}=0.224]$. No statistical significant difference was observed in distribution of generalised obesity in bank employees with respect to gender $[p=0.024]$, religion $[p=0.320]$ and education $[p=$ 0.207] (Table 4).

There was no statistical significant difference observed in distribution of generalised obesity in bank employees with respect to bank sector $(\mathrm{p}=0.92)$, employees' post ( $\mathrm{p}$ $=0.206)$ and socioeconomic status $[\mathrm{p}=0.086]$ (Table 5).

There was a significant difference in the prevalence of obesity in age group 45 years and above than the lower age groups $(\mathrm{p}=0.008)$. We didn't found any significant difference in the abdominal obesity among males and females $(\mathrm{p}=0.85)$. No significant difference was observed in the prevalence of abdominal obesity in relation with religion $(\mathrm{p}=0.258)$, education $(\mathrm{p}=0.186)$ and bank sector $(p=0.408)$ of the employees. But a significantly higher prevalence of abdominal obesity was observed in the managers and clerks than the other workers of the banks $(p=0.023)$. Also employees of the higher socioeconomic class were having significantly higher prevalence of abdominal obesity than the employees of the lower class [p=0.009] (Table 6).

Table 4: Distribution of generalized obesity in bank employees according to age, gender, religion and education.

\begin{tabular}{|c|c|c|c|c|c|c|c|}
\hline \multicolumn{2}{|l|}{ Variable } & $\begin{array}{l}\text { Under-weight } \\
\text { No }(\%)\end{array}$ & $\begin{array}{l}\text { Normal } \\
\text { No }(\%)\end{array}$ & $\begin{array}{l}\text { Over-weight } \\
\text { No }(\%)\end{array}$ & $\begin{array}{l}\text { Obese } \\
\text { No }(\%)\end{array}$ & $\begin{array}{l}\text { Total } \\
\text { No }(\%)\end{array}$ & $\begin{array}{l}\text { Statistical } \\
\text { test }\end{array}$ \\
\hline \multirow{4}{*}{$\begin{array}{l}\text { Age } \\
\text { groups } \\
\text { (years) }\end{array}$} & $25-34$ & $5(5.6)$ & $22(24.4)$ & $41(45.6)$ & $22(24.4)$ & $90(37.5)$ & \multirow{4}{*}{$\begin{array}{l}\text { Chi-Square } \\
\text { Test: } \\
\chi^{2}=6.027, \mathrm{df} \\
=4, \mathrm{p}=0.224\end{array}$} \\
\hline & $35-44$ & $2(4.8)$ & $7(16.7)$ & $20(47.6)$ & $13(31.0)$ & $42(17.5)$ & \\
\hline & $\geq 45$ & $2(1.9)$ & $16(14.8)$ & $54(50.0)$ & $36(33.3)$ & $108(45.0)$ & \\
\hline & Total & $9(3.8)$ & $45(18.8)$ & $115(47.9)$ & $71(29.6)$ & $240(100)$ & \\
\hline \multirow{3}{*}{ Gender } & Male & $8(4.0)$ & $40(20.0)$ & $100(50.0)$ & $52(26.0)$ & $200(83.3)$ & \multirow{3}{*}{$\begin{array}{l}\text { Chi-Square } \\
\text { Test: } \chi 2= \\
7.495, \mathrm{df}=2, \\
\mathrm{p}=0.024\end{array}$} \\
\hline & Female & $1(2.5)$ & $5(12.5)$ & $15(37.5)$ & $19(47.5)$ & $40(16.7)$ & \\
\hline & Total & $9(3.8)$ & 45 (18.8) & $115(47.9)$ & $71(29.6)$ & $240(100)$ & \\
\hline \multirow{5}{*}{ Religion } & Hindu & $6(3.3)$ & $38(21.0)$ & $82(45.3)$ & $55(30.4)$ & $181(75.4)$ & \multirow{5}{*}{$\begin{array}{l}\text { Fisher exact } \\
\text { test: } \\
p=0.320\end{array}$} \\
\hline & Muslim & $0(0.0)$ & $0(0.0)$ & $6(85.7)$ & $1(14.3)$ & $7(2.9)$ & \\
\hline & Buddhism & $1(2.7)$ & $5(13.5)$ & $18(48.6)$ & $13(35.1)$ & $37(15.4)$ & \\
\hline & Others & $2(13.3)$ & $2(13.3)$ & $9(60.0)$ & $2(13.3)$ & $15(6.3)$ & \\
\hline & Total & $9(3.8)$ & $45(18.8)$ & $115(47.9)$ & $71(29.6)$ & $240(100)$ & \\
\hline \multirow{6}{*}{ Education } & $\begin{array}{l}\text { Primary/Middl } \\
\text { e school }\end{array}$ & $2(6.9)$ & $7(24.1)$ & $14(48.3)$ & $6(20.7)$ & $29(12.1)$ & \multirow{6}{*}{$\begin{array}{l}\text { Fisher exact } \\
\text { test: } \\
\mathbf{p}=\mathbf{0 . 2 0 7}\end{array}$} \\
\hline & High school & $2(5.4)$ & $8(21.6)$ & $21(56.8)$ & $6(16.2)$ & $37(15.4)$ & \\
\hline & $\begin{array}{l}\text { Intermediate or } \\
\text { post high } \\
\text { school diploma }\end{array}$ & $3(3.0)$ & 19 (18.8) & 45 (44.6) & $34(33.6)$ & $101(42.1)$ & \\
\hline & $\begin{array}{l}\text { Graduate or } \\
\text { post graduate }\end{array}$ & $2(3.2)$ & $7(11.1)$ & $32(50.8)$ & $22(34.9)$ & $63(26.3)$ & \\
\hline & $\begin{array}{l}\text { Professional or } \\
\text { Honours' }\end{array}$ & $0(0.0)$ & $4(40.0)$ & $3(30.0)$ & $3(30.0)$ & $10(4.2)$ & \\
\hline & Total & $9(3.8)$ & $45(18.8)$ & $115(47.9)$ & $71(29.6)$ & $240(100.0)$ & \\
\hline
\end{tabular}


Table 5: Distribution of generalized obesity in bank employees according to bank sector, employee post and socioeconomic status (SES).

\begin{tabular}{|c|c|c|c|c|c|c|c|}
\hline & ariable & $\begin{array}{c}\text { Under-weight } \\
\text { No }(\%)\end{array}$ & $\begin{array}{l}\text { Normal } \\
\text { No }(\%)\end{array}$ & $\begin{array}{l}\text { Over-weight } \\
\text { № }(\%)\end{array}$ & $\begin{array}{l}\text { Obese } \\
\text { No }(\%) \\
\end{array}$ & $\begin{array}{l}\text { Total } \\
\text { No }(\%)\end{array}$ & $\begin{array}{l}\text { Statistical } \\
\text { test }\end{array}$ \\
\hline \multirow{4}{*}{$\begin{array}{l}\text { Bank } \\
\text { sectors }\end{array}$} & Government banks & $4(4.0)$ & $17(17.0)$ & $50(50.0)$ & $29(29.0)$ & $100(41.6)$ & \multirow{4}{*}{$\begin{array}{l}\text { Chi-Square } \\
\text { Test: } \chi 2= \\
0.99, \mathrm{df}=4, \mathrm{p} \\
=0.92\end{array}$} \\
\hline & Cooperative banks & $2(4.3)$ & $10(21.7)$ & $22(47.8)$ & $12(26.1)$ & $46(19.2)$ & \\
\hline & Private banks & $3(3.2)$ & $18(19.1)$ & $43(45.7)$ & $30(31.9)$ & $94(39.2)$ & \\
\hline & Total & $9(3.8)$ & $45(18.8)$ & $115(47.9)$ & $71(29.6)$ & $240(100.0)$ & \\
\hline \multirow{5}{*}{$\begin{array}{l}\text { Employe } \\
\text { es' Posts }\end{array}$} & Managers & $1(2.1)$ & $9(18.8)$ & $19(39.6)$ & $19(39.6)$ & $48(20.0)$ & \multirow{5}{*}{$\begin{array}{l}\text { Chi-Square } \\
\text { Test: } \chi 2= \\
8.47, \mathrm{df}=6, \mathrm{p} \\
=0.206\end{array}$} \\
\hline & Officers & $1(2.3)$ & 7 (15.9) & $24(54.5)$ & $12(27.3)$ & $44(18.3)$ & \\
\hline & Clerks & $3(3.7)$ & $14(17.1)$ & $37(45.1)$ & $28(34.1)$ & $82(34.2)$ & \\
\hline & Attendants & $4(6.1)$ & $15(22.7)$ & $35(53.0)$ & $12(18.2)$ & $66(27.5)$ & \\
\hline & Total & $9(3.8)$ & $45(18.8)$ & $115(47.9)$ & $71(29.6)$ & $240(100.0)$ & \\
\hline \multirow{4}{*}{$\begin{array}{l}\text { Socioeco } \\
\text { nomic } \\
\text { status }\end{array}$} & Upper (I) & $1(2.0)$ & $9(17.6)$ & $24(47.1)$ & $17(33.3)$ & $51(21.2)$ & \multirow{4}{*}{$\begin{array}{l}\text { Chi-Square } \\
\text { Test: } \chi 2= \\
8.14, \mathrm{df}=4, \mathrm{p} \\
=0.086\end{array}$} \\
\hline & Upper middle (II) & $4(3.3)$ & $20(16.3)$ & $56(45.5)$ & $43(35.0)$ & $123(51.2)$ & \\
\hline & Lower middle (III) & $4(6.1)$ & $16(24.2)$ & $35(53.0)$ & $11(16.7)$ & $66(27.6)$ & \\
\hline & Total & $9(3.8)$ & $45(18.8)$ & $115(47.9)$ & $71(29.6)$ & $240(100.0)$ & \\
\hline
\end{tabular}

Table 6: Distribution of abdominal obesity in bank employees according to sociodemographic variables.

\begin{tabular}{|c|c|c|c|c|c|}
\hline \multirow{2}{*}{ Variable } & & \multicolumn{2}{|c|}{ Abdominal obesity } & \multirow[b]{2}{*}{$\begin{array}{l}\text { Total } \\
\text { No. } \%\end{array}$} & \multirow[b]{2}{*}{ Statistical test } \\
\hline & & $\begin{array}{l}\text { Present } \\
\text { No. } \%\end{array}$ & $\begin{array}{c}\text { Absent } \\
\text { No. \% }\end{array}$ & & \\
\hline \multirow{4}{*}{$\begin{array}{l}\text { Age groups } \\
\text { (In Years) }\end{array}$} & $25-34$ & $49(54.4)$ & $41(45.6)$ & $90(37.5)$ & \multirow{4}{*}{$\begin{array}{l}\text { Chi-Square Test: } \\
\chi 2=9.551, \mathrm{df}=2 \\
\mathrm{p}=0.008\end{array}$} \\
\hline & $35-44$ & $22(52.4)$ & $20(47.6)$ & $42(17.5)$ & \\
\hline & $\geq 45$ & $79(73.1)$ & $29(26.9)$ & $108(45.0)$ & \\
\hline & Total & $150(62.5)$ & $90(37.5)$ & $240(100.0)$ & \\
\hline \multirow{3}{*}{ Gender } & Male & $124(62.0)$ & $76(38.0)$ & $200(83.3)$ & \multirow{3}{*}{$\begin{array}{l}\text { Chi-Square Test with Yate's } \\
\text { correction: } \\
\chi 2=0.128, \mathrm{df}=1, \mathrm{p}=0.85\end{array}$} \\
\hline & Female & $26(65.0)$ & $14(35.0)$ & $40(16.7)$ & \\
\hline & Total & $150(62.5)$ & $90(37.5)$ & $240(100.0)$ & \\
\hline \multirow{5}{*}{ Religion } & Hindu & $111(61.3)$ & $70(38.7)$ & $181(75.4)$ & \multirow{5}{*}{$\begin{array}{l}\text { Fisher Exact Test: } \\
p=0.258\end{array}$} \\
\hline & Muslim & $6(85.7)$ & $1(14.3)$ & $7(2.9)$ & \\
\hline & Buddhism & $26(70.3)$ & $11(29.7)$ & $37(15.40$ & \\
\hline & Others & $7(46.7)$ & $8(53.3)$ & $15(6.3)$ & \\
\hline & Total & $150(62.5)$ & $90(37.5)$ & $240(100.0)$ & \\
\hline \multirow{6}{*}{ Education } & Primary/middle school & $14(48.3)$ & $15(51.7)$ & $29(12.1)$ & \multirow{6}{*}{$\begin{array}{l}\text { Chi-Square Test: } \\
\chi 2=6.17, \mathrm{df}=4 \\
\mathrm{p}=0.186\end{array}$} \\
\hline & High school & $19(51.4)$ & $18(48.6)$ & $37(15.4)$ & \\
\hline & $\begin{array}{l}\text { Intermediate/post high } \\
\text { school diploma }\end{array}$ & $68(67.3)$ & $33(32.7)$ & $101(42.1)$ & \\
\hline & Graduate or PG & $42(66.7)$ & $21(33.3)$ & $63(26.3)$ & \\
\hline & Professional or honours & $7(70.0)$ & $3(30.0)$ & $10(4.2)$ & \\
\hline & Total & $150(62.5)$ & $90(37.5)$ & $240(100.0)$ & \\
\hline \multirow{4}{*}{ Bank sector } & Government & $63(63.0)$ & $37(37.0)$ & $100(41.6)$ & \multirow{4}{*}{$\begin{array}{l}\text { Chi-Square Test: } \\
\chi 2=1.79, \mathrm{df}=2 \\
\mathrm{p}=0.408\end{array}$} \\
\hline & Cooperative & $25(54.3)$ & $21(45.7)$ & $46(19.2)$ & \\
\hline & Private & $62(66.0)$ & $32(34.0)$ & $94(39.2)$ & \\
\hline & Total & $150(62.5)$ & $90(37.5)$ & $240(100.0)$ & \\
\hline \multirow{5}{*}{$\begin{array}{l}\text { Employees } \\
\text { posts }\end{array}$} & Manager & $34(70.8)$ & $14(29.2)$ & $48(20.0)$ & \multirow{5}{*}{$\begin{array}{l}\text { Chi-Square Test: } \\
\chi^{2}=9.54, \mathrm{df}=3 \\
\mathrm{p}=0.023\end{array}$} \\
\hline & Officers & $26(59.1)$ & $18(40.9)$ & $44(18.3)$ & \\
\hline & Clerks & $58(70.7)$ & $24(29.3)$ & $82(34.2)$ & \\
\hline & Attendants & $32(48.5)$ & $34(51.5)$ & $66(27.5)$ & \\
\hline & Total & $150(62.5)$ & $90(37.5)$ & $240(100.0)$ & \\
\hline \multirow{4}{*}{ SES } & Upper (I) & $36(70.6)$ & $15(29.4)$ & $51(21.2)$ & \multirow{4}{*}{$\begin{array}{l}\text { Chi-Square Test: } \\
\chi 2=9.52, \mathrm{df}=2, \mathrm{p}=0.009\end{array}$} \\
\hline & Upper middle (II) & $83(67.5)$ & $40(32.5)$ & $123(51.2)$ & \\
\hline & Lower middle (III) & $31(47.0)$ & $35(53.0)$ & $66(27.6)$ & \\
\hline & Total & $150(62.5)$ & $90(37.5)$ & $240(100.0)$ & \\
\hline
\end{tabular}


Mean ages of overweight and obese bank employees were 42.38 years and 43.01 years respectively whereas mean ages of underweight and normal weight bank employees were 36.89 years and 39.02 years respectively. The difference in mean ages of bank employees according to BMI classification was not statistically significant, $\mathrm{p}=0.142$ (Figure 1 ).

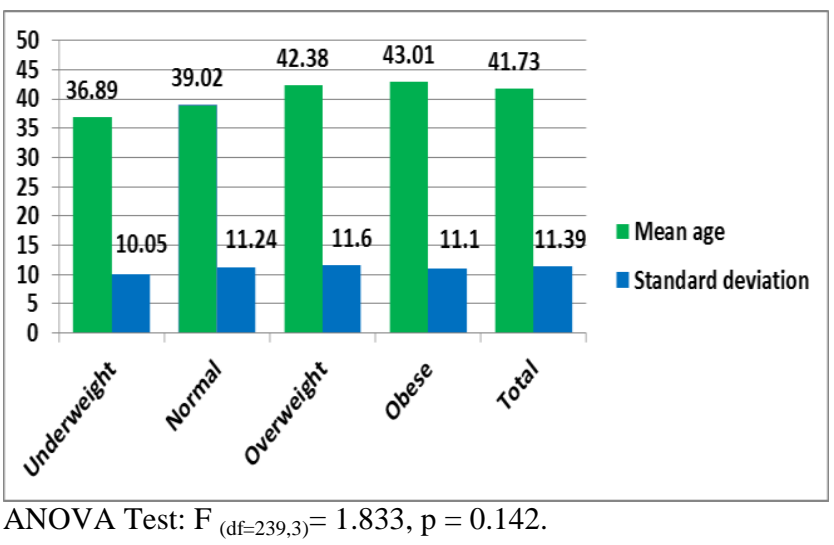

Figure 1: Age differences in bank employees by BMI classification.

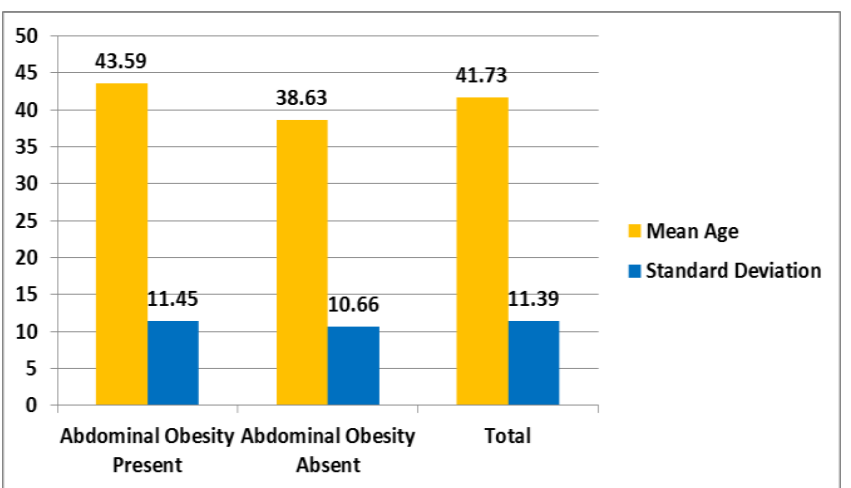

Independent samples student $\mathrm{t}$ test: $\mathrm{t}(\mathrm{df}=238)=3.332, \mathrm{p}=$ 0.001 .

Figure 2: Age difference in bank employees by abdominal obesity (waist circumference).

The mean age of bank employees who had abdominal obesity was significantly higher (43.59 years) than mean age of those who had no abdominal obesity (38.63 years), $\mathrm{p}=0.001$ (Figure 2).

\section{DISCUSSION}

In this study out of total 240 bank employees, $47.9 \%$ and $29.6 \%$ bank employees were found to be overweight and obese (GO) respectively as per BMI classification for Asian population and Overall $62.5 \%$ and $65.0 \%$ of bank employees had abdominal obesity as per waist circumference and waist-hip ratio cut off points respectively. Addo $\mathrm{P}$ et al reported overall prevalence of obesity and overweight among the bank employees was $17.8 \%$ and $37.8 \%$ respectively. ${ }^{13}$ Similarly Nagammanavar $\mathrm{R}$ et al noted $50.6 \%$ prevalence of generalized obesity as per BMI classification for Asian population and $37.65 \%$ prevalence of abdominal obesity (WHR > 0.90 in males and WHR > 0.80 in females) in bank employees. ${ }^{17}$ Assudani $\mathrm{A}$ et al found $41 \%$ of subjects were obese and $20 \%$ were overweight as per BMI classification for Asian population and $47.46 \%$ bank employee were having abdominal obesity. ${ }^{18}$ Rao V and Rao P reported the prevalence of generalized obesity was $4.3 \%$ in bank employees as per WHO International BMI classification and $19.8 \%$ prevalence of abdominal obesity in them. ${ }^{19}$ Ismail IM et al noted $62 \%$ prevalence of abdominal obesity (WHR $=0.95$ in males and $=0.85$ in females). ${ }^{20}$ Maroof KA et al found $30.0 \%$ prevalence of abdominal obesity (Waist circumference $>102 \mathrm{~cm}$ for males and $>88 \mathrm{~cm}$ for females) among bank employees. ${ }^{21}$ The variations in generalised obesity prevalence in bank employees may be due to geographical or dietary pattern differences. Similarly the variations in abdominal obesity prevalence in bank employees may be due to geographical or dietary pattern differences and/or different cut off points of WC and WHR for measuring abdominal obesity.

In our study, mean ages of overweight and obese bank employees were 42.38 years and 43.01 years respectively whereas mean ages of underweight and normal weight bank employees were 36.89 years and 39.02 years respectively. The age ranged from 25 to 60 .

Years with highest employees (45\%) belonged to the age group of 45 years and above followed by $37.5 \%$ in the age of 25 to 35 years. The differences in mean ages were not statistically significant, $\mathrm{p}=0.142$ (Table 1, Figure 1). The mean age of bank employees who had abdominal obesity was significantly higher (43.59 years) than mean age of those who had no abdominal obesity (38.63 years), $\mathrm{p}=0.001$ (Figure 2). Addo $\mathrm{P}$ et al in their study found age of bank employees ranged from 19 to 54 years, The difference in age wise distribution of generalised obesity was found statistically significant, $\mathrm{p}=0.001, \mathrm{p}<0.0001$. $^{13}$ Assudani A et al found that most of the bank employees $(69 \%)$ belonged to the age range of $26-35$ years. ${ }^{18}$

Lokare $\mathrm{L}$ et al reported $22 \%, 40 \%$, and $38 \%$ bank employees belonged to 30 - 40, 40 - 50, and 50-60 years of age groups respectively. ${ }^{22}$

The present study findings of age distribution according to generalised and abdominal obesity are not similar to these studies. This may be because of different inclusion and exclusion criteria used in various studies.

We did not found any significant difference in the generalised obesity among different age groups $(p=0.224)$ but there was a significant difference in the abdominal obesity with more prevalence in the higher age group of $>$ 45 years $(p=0.008)$. A significant difference was observed in the prevalence of generalised obesity among male and female $(\mathrm{p}=0.024)$ while gender wise no significant difference was observed in the prevalence of 
abdominal obesity $(\mathrm{p}=0.85)$ (Table 5 and 6). The present study's finding of bank employees are similar to previous studies done by Nagammanavar R et al, Maroof KA et al and Rao V and Rao P. ${ }^{17,19,21}$ But the sex wise distribution of the present study is not comparable to Addo P et al and Assudani A et al. ${ }^{13,18}$

The prevalence of generalised obesity in both male and female bank employees found in the present study is more than in Addo $\mathrm{P}$ et al. ${ }^{13}$ Whereas the prevalence of generalised obesity in Shivaramakrishna HR et al is more in male and less in female than of the present study. ${ }^{23}$ Furthermore prevalence of abdominal obesity in both sex is more than of Shivaramakrishna HR et al. ${ }^{23}$

The prevalence of either generalised obesity or abdominal obesity did not showed any significant difference in different religions, various education status and bank sectors of the employees. There was more prevalence of generalised obesity in the bank employees requiring sedentary jobs like managers, officers and clerks than the others but this difference was not significant $(\mathrm{p}=0.206)$. But significantly higher prevalence of abdominal obesity was observed in these employees $(\mathrm{p}=0.023)$ (Table 5 and 6).

Education wise findings of present study were partially comparable with the studies done by Adoo P et al, Nagammanavar R et al, and Ismail IM et al. ${ }^{13,1720}$

The present study findings of employees' posts were partially comparable to studies by Nagammanavar R et al Ismail IM et al and Momin MH et al. ${ }^{17,20,24}$

When the prevalence of generalised obesity and abdominal obesity was compared with respect to socioeconomic status of the employee, it was found that employees belonging to upper socioeconomic status were having more prevalence of generalised obesity which was not significant $(\mathrm{p}=0.086)$ while employees of upper socioeconomic status were having significantly more prevalence of abdominal obesity $(\mathrm{p}=0.009)$ than the employee of lower socioeconomic status (Table 5 and 6).

Nagammanavar $\mathrm{R}$ et al noted that $78.2 \%, 15.6 \%$, and $6.2 \%$ were of upper class, upper middle class and lower middle class socioeconomic status respectively. ${ }^{17}$

Ismail IM et al found in their study that out of 117 bank employees, $67.5 \%, 23.1 \%$ and $9.4 \%$ were of upper class, upper middle class and lower middle class socioeconomic status respectively. ${ }^{21}$ Lokare $\mathrm{L}$ et al noted that $5.0 \%$, $85 \%$, and $10 \%$ were of upper class, upper middle class and lower middle class socioeconomic status respectively. $^{22}$ Momin $\mathrm{MH}$ et al found that $70.46 \%$, $21.3 \%$ and $8.24 \%$ were of upper class, upper middle class and lower middle class socioeconomic status respectively. ${ }^{24}$ The socioeconomic status distribution of bank employees in the present study was partially comparable to the above mentioned studies as the selection criteria of banks and studies themselves may differ. The socioeconomic wise prevalence of obesity among bank employees were not mentioned in studies reviewed.

\section{CONCLUSION}

In the present study the prevalence of generalized as well abdominal obesity was very high among bank employees. The associated risk factors which were found to be significant were age, female gender, higher socioeconomic status, and higher employees' posts.

\section{Funding: No funding sources}

Conflict of interest: None declared

Ethical approval: The study was approved by institutional ethics committee of Government Medical College \& Hospital, Aurangabad, Maharashtra, India

\section{REFERENCES}

1. Shetty P. Food and nutrition: Diet, lifestyles, and obesity. In: Detels Roger, JamesMcEwen, Robert Beaglehole HT, editor. Oxford Textbook of Public Health. 4th ed.Oxford University Press; 2002. p. 353-4.

2. World Health Organization (WHO). Global status report on non-communicable diseases 2014. World Health Organization. Switzerland; 2014.

3. Seidell JC, Halberstadt J. The global burden of obesity and the challenges of prevention. Ann Nutr Metab. 2015;66Suppl 2(Suppl. 2):7-12.

4. Lim S, Vos T, Flaxman A, Danaei G, Shibuya K, Adair-Rohani $\mathrm{H}$, et al. A comparative risk assessment of burden of disease and injury attributable to 67 risk factors and risk factor clusters in 21 regions, 1990-2010: a systematic analysis for the Global Burden of Disease Study 2010. Lancet. 2012;380(9859):2224-60.

5. Pradeepa R, Anjana RM, Joshi SR, Bhansali A, Deepa M, Joshi PP. Prevalence of generalized \& abdominal obesity in urban \& rural-the ICMRINDIA B Study (PhaseI) [ICMR-INDIAB-3]. Indian J Med Res. 2015;142:139-50.

6. Shukla HC, Gupta PC, Mehta HC, Hebert JR. Descriptive epidemiology of body mass index of an urban adult population in western India. J Epidemiol Community Health. 2002;56(11):876-80.

7. Dhanaraj E, Bhansali A, Jaggi S, Dutta P, Jain S, Tiwari P, et al. Predictors of metabolic syndrome in Asian north Indians with newly detected type 2 diabetes. Indian J Med Res. 2009;129:506-14.

8. Dinsa GD, Goryakin Y, Fumagalli E, Suhrcke M. Obesity and socioeconomic status indeveloping countries: a systematic review. Obes Rev. 2012;13(11):1067-79.

9. Ahluwalia G. Obesity paradigm in India: Are the consequences an impending Tsunami? Indian J Med Res. 2015;142(2):106-8. 
10. Jogunola OO, Awoyemi AO. Prevalence of Sedentary Lifestyle among Bankers in IlorinMetropolis. Niger J Med Rehabil. 2012;15(1 and 2):44-50.

11. Owen N, Healy GN, Matthews CE, Dunstan DW. Too much sitting: the population health science of sedentary behavior. Exerc Sport Sci Rev. 2010; 38(3):105-13.

12. Koshy JM, Johnson WMS, Priya BS. Sedentary work-a risk factor for diabetes with a positive family history. Int J Sci Res. 2015;4(9):812-9.

13. Addo P, Nyarko K, Sackey S, Akweongo P, Sarfo B. Prevalence of obesity and overweight and associated factors among financial institution workers in Accra Metropolis, Ghana: a cross sectional study. BMC Res Notes BioMed Central Ltd. 2015;8(1):599.

14. World Health Organization: Western Pacific Region and International Association for the Study of Obesity (IASO). The Asia-Pacific perspective: redefining obesity and its treatment. Health Communications Australia Pty Limited; 2000.

15. WHO Expert Consultation. Waist Circumference and Waist-Hip Ratio: Report of a WHO Expert Consultation Geneva, 8-11 December 2008. World Health Organization. 2008.

16. Oberoi S. Updating income ranges for Kuppuswamy's socio-economic status scale for the year 2014. Indian J Public Health. 2015;59(2):1567.

17. Nagammanavar R, Somashekhar G, Reddy C, Kumar P, Raghavendra B. A Study of Prevalence and Risk Factors of Hypertension Among the Bank Employees of Bellary City: a Cross-Sectional Study. J Sci. 2015;5(7):459-66.
18. Assudani A, Sheth M, Jain N, Parnami S. Indirect determinant's of obesity in bankemployees of urban vadodara - a cross sectional study. Int J Appl Biol Pharm Technol. 2014;5(3):5-12.

19. Rao V, Rao P. High prevalence of coronary risk factors among bank employees in India. Int J Med Public Heal. 2014;4(4):500-3.

20. Ismail I, Kulkarni A, Kamble S, Rekha R, Amruth M, Borker S. Prevalence of hypertension and its risk factors among bank employees of Sullia Taluk, Karnataka. Sahel Med J. 2013;16(4):139-43.

21. Maroof KA, Parashar P, Bansal R, Ahmad S. A study on hypertension among the bank employees of Meerut district of Uttar Pradesh. Indian J Public Health. 2007;51(4):225-7.

22. Lokare L, Nekar MS, Mulkipatil SY, Venktesh M. Original article Metabolic Equivalent Task Score and Risk Factors of Coronary Heart Disease in Bank Employees. Int J Biol Med Res. 2012;2(4):1627-30.

23. Shivaramakrishna HR, Wantamutte S, Sangolli HN. Risk Factors of Coronary Heart Disease among Bank Employees of Belgaum City - Cross-Sectional Study. J Med. 2010;3(2):152-9.

24. Momin MH, Desai VK, Kavishwar AB. Study of socio-demographic factors affecting prevalence of hypertension among bank employees of Surat City. Indian J Public Health. 2012;56(1):44-8.

Cite this article as: Hirani S, Kuril BM, Lone DK, Ankushe RT, Doibale MK. Obesity prevalence and its relation with some sociodemographic factors in bank employee of Aurangabad city, Maharashtra, India. Int J Community Med Public Health 2016;3:1628-35. 